\title{
Transposition
}

Musique et Sciences Sociales

En lutte | 2020

Sciences en danger, revues en lutte

\section{La ciencia en peligro, las revistas en lucha}

\section{Collectif des revues en lutte y Camille Noûs}

Traductor. Julia Chardavoine y Maria Teresa Martinez Trujillo

\section{CpenEdition}

\section{Journals}

Edición electrónica

URL: http://journals.openedition.org/transposition/5356

DOI: 10.4000/transposition.5356

ISSN: 2110-6134

Editor

CRAL - Centre de recherche sur les arts et le langage

\section{Referencia electrónica}

Collectif des revues en lutte et Camille Noûs, «La ciencia en peligro, las revistas en lucha »,

Transposition [En ligne], En lutte | 2020, mis en ligne le 09 mars 2020, consulté le 24 septembre 2020

URL : http://journals.openedition.org/transposition/5356 ; DOI : https://doi.org/10.4000/transposition. 5356

Este documento fue generado automáticamente el 24 septiembre 2020

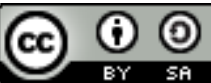

La revue Transposition est mise à disposition selon les termes de la Licence Creative Commons Attribution - Partage dans les Mêmes Conditions 4.0 International 


\title{
La ciencia en peligro, las revistas en lucha
}

\author{
Collectif des revues en lutte y Camille Noûs \\ Tradución : Julia Chardavoine y Maria Teresa Martinez Trujillo
}

\section{NOTA DEL EDITOR}

Editorial común del Colectivo de Revistas en Lucha, consultable en línea en Université Ouverte (https://universiteouverte.org/2020/03/05/edito-commun). Hasta el 9 de marzo 2020, dos meses después del inicio de la movilización, 147 revistas se han sumado al Colectivo de Revistas en Lucha. La lista de revistas se puede consultar en universiteouverte.org

\section{Revues en lutte}

1 Desde principios de 2020, más de cien revistas académicas francesas, de ciencias humanas y sociales en su gran mayoría, se han declarado "en lucha" o "en huelga". Sus comités de redacción participan en el actual movimiento social, en el que se protesta en contra del plan para las pensiones, contra la reforma del seguro de desempleo adoptada en otoño de 2019, y contra las propuestas que figuran en los informes para la Ley de Programación Multianual de la Investigación (LPPR por sus siglas en francés) ${ }^{1}$. Dada su magnitud y su forma - la huelga y el voto de mociones que alientan los comités de redacción a exponerse más que de costumbre -, esta movilización no tiene precedentes históricos. La dinámica colectiva que genera, más allá de las disciplinas, de las escuelas y de las condiciones en que ejerce cada una de las revistas, refleja la indignación provocada por estas reformas. Para la educación superior y la investigación, la reforma de las pensiones, según lo previsto actualmente por el gobierno, será responsable del aumento general de las desigualdades (entre hombres y mujeres, entre personal titular y trabajadores en condiciones precarias es decir pagados por honorarios, etc.) y del empobrecimiento de todas y todos, ya sean funcionarios públicos, empleados con 
contrato o en condiciones precarias. La reforma del seguro de desempleo también aumentará la vulnerabilidad, de por sí difícil de soportar, del gran número de trabajadores precarios de quienes depende, en gran medida, el buen funcionamiento de las universidades y centros de investigaciones: ellos y ellas representan más de una cuarta parte del personal docente, y una proporción aún mayor entre los trabajadores administrativos y técnicos. Finalmente, la LPPR solo empeorará la escasez de recursos, empleos y estabilidad, y profundizará las desigualdades que en dos décadas de "reformas", masivamente impugnadas, no han dejado de dañar la educación superior y la investigación.

\section{Una crisis planeada del servicio público de investigación y de las universidades}

2 Desde hace casi treinta años, los sucesivos gobiernos han contribuido a la erosión del Estado social, al lento recorte del servicio público, a regatear los "privilegios" ganados durante las luchas sociales del siglo XX, así como al debilitamiento de los principios de redistribución, cuya finalidad es regular las disparidades socioeconómicas y geográficas. En la educación superior y la investigación, la ley relativa a las libertades y responsabilidades de las universidades, conocida como la "Ley Pécresse" (LRU, 2007) se convirtió en la piedra angular de un doble movimiento, aparentemente contradictorio: por un lado, de acuerdo con la lógica neoliberal, el Estado reduce su participación presupuestal, pero, por otro lado, se encarga autoritariamente de la conducción estratégica de la investigación. La proclamada autonomía (presupuestaria) oculta la desregulación de los estatutos laborales, la competencia de tod@s contra tod@s y la creciente dependencia de la investigación en los intereses económicos e industriales, lo que en última instancia cuestiona su verdadera autonomía. Esta política, llevada a cabo con obstinación y desafiando las advertencias y reclamaciones de la comunidad investigadora, dio lugar a la creación de numerosas agencias de evaluación y financiamiento, supuestamente independientes, que abogan por una cultura de "desempeño", "resultado" y "excelencia", mientras reducen los presupuestos propios de los centros de investigaciones a favor de una distribución selectiva de recursos definida, en gran medida, por las fluctuaciones coyunturales (si no las tendencias) de la economía, así como por las jerarquías y otras situaciones preestablecidas. Al favorecer el financiamiento por proyectos, esta política ha aumentado la desigualdad entre investigadores y ha llevado a un inmenso desperdicio de energía y dinero público: ¿cuántas horas se pierden en evaluar o escribir proyectos para obtener un financiamiento hipotético, mientras este tiempo podría haberse dedicado a la investigación o docencia?

3 Sólo por poner un ejemplo, los "gobernantes" alimentan una obsesión mórbida con las clasificaciones internacionales, cuya razón de ser es la promoción del supuesto modelo anglosajón de administración de las universidades como si se tratara de empresas, es decir que operan con sus propios fondos (nutridos por las cuotas de inscripción que no cesan de aumentar), aunque eso signifique sacrificar el presupuesto operativo y la calidad de la docencia. Desde la LRU, a menudo se invoca la supuesta mala ubicación de las universidades francesas en los rankings internacionales, y a partir de ello se profundiza, contra la voluntad de la comunidad investigadora, la liberalización de la educación superior y de la investigación. Sin embargo, estos mandatos se implementan 
en un contexto de fuerte austeridad presupuestaria: en comparación con el número de estudiantes, el presupuesto designado a la educación superior ha caído en más del10\% desde 2010; y a pesar de que durante veinte años los sucesivos gobiernos han repetido la promesa de incrementar el presupuesto dedicado a la investigación pública para alcanzar el 1\% del PIB, éste permanece estancado en el $0.8 \%$ (es decir, hacen falta seis mil millones de euros para alcanzar tal meta, una suma inferior al crédito fiscal de investigación, nicho que ha sido otorgado a grandes grupos industriales y de servicios). En estas condiciones, los investigadores y académicos en Francia están sujet@s a un régimen que combina un exceso de trabajo con el deterioro de las condiciones laborales y de vida. Tienen cada vez empleos más precarios y por más tiempo; hoy la edad promedio de reclutamiento a un puesto de titular es de 35 años. Las políticas de austeridad también conducen a una reducción drástica de sus ingresos: mientras que, en treinta años, los titulares han visto caer su poder adquisitivo en un $30 \%$ - lo que hace una gran diferencia respecto al sector privado -, los trabajadores temporales son aún más vulnerables, acumulan contratos cortos y períodos de incertidumbre, y aceptan simultáneamente varios contratos como temporales, los cuales son pagados por debajo del salario mínimo por hora. Cada vez más se evalúa a investigadores y docentes a partir de criterios estrictamente contables que se limitan a contar sus publicaciones, contratos obtenidos o patentes registradas, sin cuestionar la contribución real que el conocimiento producido supone para la ciencia. Estas distintas lógicas favorecen el surgimiento de "empresarios de carrera", en detrimento de la investigación fundamental, colectiva y verdaderamente independiente.

4 Las medidas anunciadas en el marco de la LPPR continúan obstinadamente las transformaciones neoliberales iniciadas desde principios de la década de 2000, a raíz de la Agenda de Lisboa preparada por el Consejo Europeo, e incluso buscan radicalizarlas. Así, las consignas pertenecen al léxico gerencial: competitividad, financiamiento de proyectos, concentración desigual de recursos o austeridad presupuestaria, términos que conducen al desarrollo de trabajos precarios y a la competencia entre individuos, centros de investigaciones, establecimientos, etc. Como lo dijo claramente, en noviembre de 2019, el director general del CNRS, Antoine Petit se trata de iniciar una reforma "desigual y darwiniana": generalizar la competencia y concentrar los recursos en una minoría de establecimientos e individuos, considerados como los más "eficientes" según criterios de gestión. Éstos se convierte en los principios cardinales de la dirección de la educación superior y de la investigación en un contexto de "desafíos sociales" muy permeables a las prioridades de la acción gubernamental. Sin embargo, numerosas encuestas demuestran los efectos nocivos de tales políticas sobre la originalidad del conocimiento producido y sobre la calidad de la formación impartida a las nuevas generaciones: el "efecto Mathieu" - el proceso por el cual los más favorecidos incrementan su ventaja -, la estandarización de la investigación, la burocratización, el debilitamiento de la autonomía universitaria, el empobrecimiento de la diversidad disciplinaria, etc.

5 Toda esta política desprecia lo que los investigadores han aprendido de la experiencia: en todas las disciplinas, la actividad científica requiere tiempo y disponibilidad intelectual, la cual es incompatible con la ansiedad que implica la precariedad, a veces extrema, y con la creciente fragmentación de las tareas; se ejerce mucho mejor cuando los equipos están unidos mientras que la competencia entre pares, ahora exacerbada, amenaza a los colectivos; requiere también una distancia crítica que hoy se ve obstaculizada por la dependencia de las jerarquías administrativas. Por lo tanto, la 
comunidad investigadora exige firmemente un compromiso presupuestario para enfrentar los desafíos (logrando al menos el objetivo del 1\% del PIB dedicado a la investigación pública) y una distribución equitativa de los recursos entre el personal titular, cuyo estatus de funcionario público sigue siendo la condición para la independencia y sinceridad de los resultados que obtiene.

\section{Revistas científicas: una economía del conocimiento eficaz}

6 En este contexto, nuestras revistas científicas tienen un papel singular y paradójico. Al ser lugares de intenso trabajo colectivo de producción y soporte eficaces para la difusión del conocimiento, las revistas tienden a instrumentalizarse y ponerse al servicio de la visión dominante neo-gerencial de la investigación. El proceso de evaluación de los investigadores, centros de investigaciones y universidades ahora se basa, en gran medida, en un recuento de los artículos publicados en nuestras revistas, de acuerdo con cálculos bibliométricos, cuya debilidad y efectos perversos en el plano científico ya han sido ampliamente documentados ${ }^{2}$. Esta no es una paradoja menor de las reformas en curso: si bien colocan a las revistas, más que nunca, en el corazón de este sistema de investigación, regido por la "excelencia" bibliométrica, también debilitan su funcionamiento y desvirtúan su trabajo de producción científica.

7 Es por eso que, al perturbar o suspender nuestra actividad, nos rehusamos a permanecer distantes de lo que afecta tanto a la comunidad científica como al mundo social $\mathrm{y}$, al hacerlo, deseamos resaltar tanto lo que se hace en las revistas, como a los y las que las hacen. Porque nuestro trabajo colectivo, intelectual y editorial, que permite la producción y el intercambio de conocimientos, está siendo directamente amenazado por los proyectos de ley actuales, que debilitan cada vez más el servicio público de educación superior e investigación.

La existencia de nuestras revistas se integra a una economía del conocimiento frágil, pero eficaz. Son los propios científic@s, muchos de los cuales son funcionari@s públic@s, quienes, a partir de minuciosos análisis, evalúan los textos, los discuten, los aceptan o no, hacen sugerencias a sus autores para que los textos sean más relevantes, más completos, más exigentes en su demostración, y que, al final de este largo proceso de revisiones, discusiones, reescrituras, deliberaciones colectivas e intercambios con les autores, se publiquen y difundan, en forma de artículos científicos, trabajos que puedan contribuir al conocimiento colectivo. Estos textos también se benefician del detallado trabajo de verificación formal, formateo y publicación en línea llevado a cabo, si no por los investigadores, por profesionales de la documentación, edición y/o del manejo de plataformas digitales que laboran en condiciones diversas, e ya sea más o menos precarias - desde funcionarios públicos hasta trabajadores con contrato temporales, pasando por los micro-emprendedores-. Finalmente, son principalmente las bibliotecas universitarias y los organismos públicos quienes compran estas revistas, ya sea por separado o en paquete a través de plataformas digitales. Esta oferta en línea, gratuita para los estudiantes, docentes e investigadores, e incluso para tod@s cuando se trata de revistas de libre acceso en Internet, permite una amplia difusión de los últimos avances científicos fuera del campo universitario. Gracias al trabajo paciente y colectivo de las revistas académicas, los docentes, los periodistas, las asociaciones, los funcionari@s 
electos así como les ciudadan@s tienen acceso a un conocimiento sustancial, confiable y actualizado periódicamente.

9 Sin embargo, si bien esta economía del conocimiento asegura el enriquecimiento del saber, no genera ganancias sustanciales en términos financieros. Por ello, está respaldada por una infraestructura invisible, la del servicio público de la investigación.

10 Este servicio público garantiza, idealmente, un personal capacitado, calificado y estable en los comités de redacción.

11 Este servicio público ofrece, idealmente, redes o casas editoriales para la digitalización, el archivo o la promoción de los artículos.

12 Este servicio público permite, idealmente, la existencia de revistas científicas digitales de calidad disponibles en libre acceso y completamente gratuitas.

13 Este servicio público nos brinda, finalmente, el tiempo necesario para formar parte de los comités de redacción, para concebir los números especiales, para leer, evaluar y discutir los artículos propuestos, a pesar del progresivo deterioro de las condiciones laborales del personal titular y de las precarias condiciones de trabajo de los jóvenes docentes e investigadores.

14 A pesar de todo, al final, los pocos ingresos generados por las revistas no se utilizan para remunerar ni a 1@s científic@s que las nutren ni a los trabajadores que las fabrican. La mayor parte de estos ingresos se destina a las empresas que distribuyen estas revistas en plataformas de publicación científica, privadas en su mayoría, dentro de un frágil sector editorial. A nivel internacional y en todas las disciplinas científicas más allá de las humanidades y ciencias sociales, la situación es aún más compleja. Estas empresas obtienen importantes ventajas en el trabajo de edición: por un lado, se ahorran las tareas de revisión gracias al trabajo voluntario de 1@s académic@s a quienes solicitan esta labor; por otro lado, para financiar la práctica del acceso abierto, recurren al modelo invertido en el que "el autor / la autora paga". Por lo tanto, la comunidad científica y sus fondos públicos a menudo pagan por una actividad cuyos beneficios llegan finalmente a estas organizaciones comerciales depredadoras. Para luchar contra esta mercantilización del conocimiento, algunas plataformas y algunas revistas han ofrecido en los últimos años dispositivos de acceso abierto completo, donde el autor no paga. Estos dispositivos deben ser reforzados y apoyados financieramente por las autoridades públicas para difundir el conocimiento científico aún más ampliamente.

La LPPR, según lo que se anunció, promete socavar los fundamentos de la triple economía financiera, científica y humana de las revistas. Está afectando directamente al denominado personal de apoyo a la investigación, aquellos que garantizan la existencia de las revistas como objetos, productos manufacturados (incluso en el espacio virtual, un artículo tiene que respetar estándares tipográficos y bibliográficos precisos y un formato, así como ser editado). La reforma crea condiciones laborales precarias y sustituye los puestos titulares por contratos llamados "de sitio", lo que obligará nuestras revistas a gastar una energía tremenda para tramitar expedientes administrativos complejos y beneficiarse de unas pocas horas de trabajo de una persona especializada en la edición. Se trata de seguir una política cínica de reducción drástica de la cantidad de trabajadores que permanecen invisibles en la cadena editorial (editores, secretari@s de redacción, traductores, diseñadores gráficos, desarrolladores, impresores y emplead@s de las plataformas digitales, etc.). De hecho, entre estos 
últimos, las pocas personas que se benefician de un contrato permanente de trabajo o del estatus de funcionari@ públic@, generalmente carecen de equipos de trabajo y tienen una carga excesiva de tareas, dado el flujo constante que implica la publicación de revistas. Incluso, dado que el el trabajo atribuido a cada puesto tiende a "mutualizarse" entre diversas publicaciones, se duplica o triplica la carga de tareas asociadas a cada puesto. Aquell@s que tienen que acumular contratos mal pagados y temporales, por su parte, también se ven obligad@s a trabajar mucho más que las horas realmente pagadas, alternando entre períodos de desempleo y empleos inciertos en el seno de equipos a los que, dada su falta de capacitación, no alogran integrarse. En esta situación está el personal empleado por OpenEdition, del cual casi el $60 \%$ tiene un contrato temporal o de prestación de servicios profesionales (lo que equivaldría a contrato por honorarios), mientras que la plataforma se ha vuelto indispensable para la mayoría de nuestras revistas ${ }^{3}$. Ya conocemos las consecuencias de este sistema que recuerdan lo que se evidenció en el caso, en particular, de France-Télécom, La Poste o de los hospitales públicos: exceso de trabajo, deterioro de las condiciones laborales y de los estatutos, lo cual genera en los trabajadores malestar, incertidumbre permanente, pérdida de sentido de su labor y sensación de desperdicio de sus competencias profesionales.

No solamente la LPPR favorece la investigación por proyectos y los contratos temporales, reduciendo drásticamente la cantidad de investigadores titulares, además, fragiliza dramáticamente la posición de los investigadores jóvenes en búsqueda de trabajo. Estos se ven obligad@s a aceptar varios contratos posdoctorales de tiempo definido para vivir o bien, a salir de Francia para ir a donde se les ofrecen los puestos que faltan aquí, o incluso abandonar la investigación para incursionar en otro tipo de trabajo. De manera contrastante, estos jóvenes investigadores contribuyen masivamente a la producción de artículos científicos y a la renovación del conocimiento.

\section{Defender la autonomía de la investigación y de la edición científica}

Como lo hemos visto, el trabajo detrás de nuestras revistas es un esfuerzo paciente de discusión, e incluso - si se nos permite la palabra a pesar de sus usos actuales - de evaluación a partir de criterios compartidos. La transparencia y la pedagogía de los procesos editoriales, de la evaluación de artículos, de su aceptación o rechazo, tienen importantes consecuencias en las trayectorias de los investigadores y académic@s, especialmente para aquell@s que buscan un puesto de trabajo ; y el papel desempeñado por las revistas en este proceso es innegable. Pero, aunque le pese a quienes consideran nuestras revistas como herramientas por excelencia de la selección "desigual y darwiniana", no son agencias de calificación destinadas a establecer el ranking de investigadores, a clasificar los "talentos" o a medir los "rendimientos". Porque el proceso de evaluación es colegiado, arbitrado a partir de la deliberación colectiva, que busca producir conocimientos más precisos, robustos y mejor demostrados. Si no se aceptan todos los artículos que se envían, no es porque nuestras revistas son instancias de eliminación con criterios de "excelencia" definidos por alguna burocracia; en cambio, es porque son lugares de reflexión y apreciación, pero también de diálogo con los autores, para definir lo que, con respecto a sus proyectos intelectuales, contribuye a 
"hacer ciencia". Ya sean generales, especializadas o interdisciplinarias, las revistas ayudan a informar a la comunidad científica - y más allá - de las investigaciones en curso, pero también a hacer nuevas preguntas, proponer nuevos análisis o interpretaciones, y provocar controversias. En el vasto ecosistema de revistas académicas, cada comité de redacción trabaja para desarrollar una línea editorial que nutra la identidad de la revista y no corresponda a una concepción homogénea de la cientificidad. Por lo tanto, basado en prácticas colectivas y en una concepción cooperativa y acumulativa de la investigación científica, el espíritu que anima nuestras revistas se opone a la competencia y a la evaluación individual ilusoria de los investigadores.

La coexistencia de diferentes revistas es por lo tanto indispensable: la pluralidad y la emulación son las condiciones del debate y de la confrontación, necesarias para el progreso y la validación del conocimiento. La ciencia se elabora sobre la contradicción y la multiplicidad de enfoques y escuelas; es precisamente lo que la concentración de recursos pone en peligro. A diferencia de la concepción gerencial, que tiende a convertir a las revistas en centros de selección y registro de una ciencia estandarizada a nivel mundial, la capacidad de las diferentes revistas (nacionales en particular) para defender un punto de vista científico particular, una línea editorial específica, permite la existencia de un espacio internacional con varios puntos de vista y diversos enfoques, lo que garantiza la dinámica de la ciencia. Por lo tanto, las revistas científicas son organismos de producción y difusión de un conocimiento certificado colectivamente. Los artículos y los números especiales que publican son el fruto de trabajos originales: en las humanidades y las ciencias sociales, meses de investigación en archivos o trabajos de campo en los que se colecta información empírica inédita pueden presentarse en 50,000 valiosos caracteres. Este proceso de evaluación, deliberación colectiva e intercambios entre los comités de redacción y los autores toma largos meses de trabajo, por lo que pocos artículos se publican en su versión inicial. Los autores, los evaluadores, los miembr@s del comité de redacción y los secretari@s de redacción contribuyen juntos a la creación de un conocimiento confiable y accesible. Nuestras revistas son lugares de transmisión, traducción y producción de ideas e investigaciones, así como espacios para encuentros y debates; garantizan un conocimiento científicamente sólido e intelectualmente libre, protegido de intereses privados. Contribuyen a mejorar la ciencia.

El mundo de la investigación ya está estructurado a partir de una aguda competencia. Si queremos fortalecer la calidad y la diversidad de la producción científica, no necesitamos al darwinismo social, sino espacios de trabajo estables y "entornos" estructurados, sin los cuales es imposible asumir riesgos, cooperar y debatir, y así producir y consolidar el conocimiento. Al imponer reformas estructurales permanentes, la conducción vertical por proyectos, procesos acelerados y concebidos a corto plazo, condiciones más precarias para los trabajadores y colectivos de trabajo, la serie de reformas, que incluye a la LPPR solo desestabiliza y empobrece el frágil ecosistema de las revistas científicas.

Ante el deterioro del servicio público de investigación, las revistas expresan su enfado y preocupación, y actúan de forma inédita: se declaran en huelga, se movilizan con el personal que lucha, publican números completamente en blanco o contribuyen, mediante la publicación de textos colectivos o relatos anónimos, al movimiento social actual. Revelan lo que hay detrás de la cortina y todo lo que hace posible la producción 
y difusión de un conocimiento independiente (especialmente de los beneficios industriales), confiable (porque es fruto del debate de científicos de alto nivel) y nuevo (este conocimiento es la base de los futuros libros de texto universitarios y escolares). Nuestras revistas deben su existencia solo al servicio público de investigación. Debido a que el servicio público en general, y el de la investigación en particular, están amenazados, nosotros, el colectivo de las revistas en lucha, nos oponemos con la mayor firmeza a los proyectos de reforma en curso. Nos negamos a destruir las formas de colaboración y emulación solidarias que son la fuerza y el honor del modelo de investigación francés.

\section{Revues en lutte}

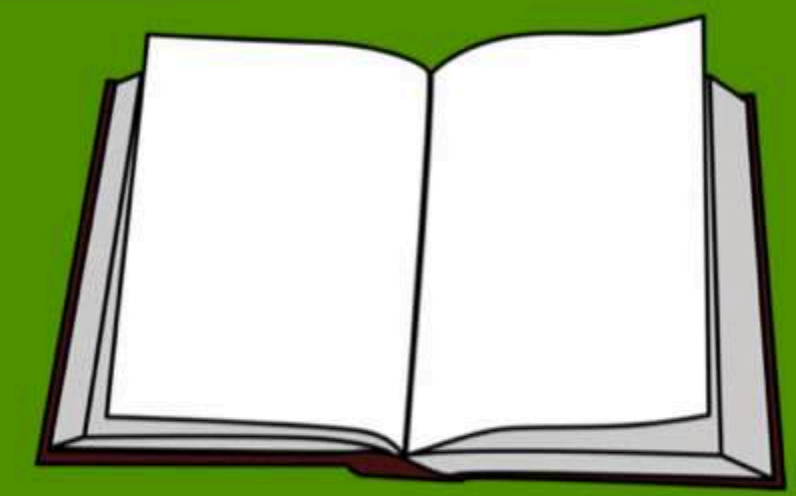

Ici, vous auriez pu lire un article scientifique de haut niveau. Malheureusement, faute de poste, son auteur a dû changer de métier avant de l'avoir écrit...

@RevuesFnLutte / com_mob_revues@framalistes.org

\section{NOTAS}

1. Los informes están firmados por tres grupos de trabajo dedicados a la "Financiación de la investigación", a la "Capacidad de atracción de los empleos y carreras científicas" y a la "Investigación asociativa e innovación". Están disponibles en el sitio web del Ministerio de Educación Superior, Investigación e Innovación: https://www.enseignementsuprecherche.gouv.fr/cid145221/restitution-des-travaux-des-groupes-de-travail-pour-un-projet-deloi-de-programmation-pluriannuelle-de-la-recherche.html

2. Yves Gingras, Les dérives de l'évaluation de la recherche. Du bon usage de la bibliométrie, Raisons d'agir, Paris, 2014.

3. Ver https://academia.hypotheses.org/13060 